\title{
Injeção intravítrea de triancinolona no tratamento da telangiectasia retiniana justafoveolar idiopática
}

\author{
Intravitreal triamcinolone injection in the treatmentofidiopathic \\ juxtafoveal telangiectasis
}

\author{
Otacílio Oliveira Maia Júnior ${ }^{1}$ \\ Walter Yukihiko Takahashi ${ }^{2}$ \\ Maria Teresa Brizzi C. Bonanomi ${ }^{3}$ \\ Vinícius Paganini Nascimento ${ }^{4}$ \\ Carlos Sérgio Nascimento de Melo ${ }^{5}$
}

\begin{tabular}{|l|}
\hline RESUMO \\
\hline Relato de caso de um paciente com telangiectasia justafoveal idiopática \\
(TJI) tipo 1A, no olho direito, submetido a $4 \mathrm{mg}$ de triancinolona \\
intravítrea. O resultado foi avaliado por meio da acuidade visual e da \\
tomografia de coerência óptica. A acuidade visual e a espessura retiniana \\
macular medida na tomografia de coerência óptica, antes da injeção \\
intravítrea de triancinolona, foram respectivamente de $20 / 100$ e $569 \mu \mathrm{m}$ e, \\
após três semanas do tratamento foram de $20 / 60$ e $371 \mu$ m e na sexta \\
semana de 20/100 e $614 \mu$ m. A estabilização da parede vascularobtida com \\
injeção intravítrea de triancinolona proporciona melhora transitória da \\
visão e do edema macular em olhos com TJI-1A. Não foi demonstrada \\
nenhuma ajuda permanente à fotocoagulação prévia.
\end{tabular}

Descritores: Fóvea central/patologia; Fundo de olho; Neovascularização retiniana; Telangiectasia/diagnóstico; Triancinolona/uso terapêutico; Tomografia de coerência óptica; Relatos de casos [tipo de publicação]

\section{INTRODUCÃO}

Trabalho desenvolvido no Setor de Retina e Vítreo do Departamento de Oftalmologia do Hospital das Clínicas da Faculdade de Medicina da Universidade de São Paulo - USP - São Paulo (SP) - Brasil.

Pós-Graduando (Nível Doutorado) da Faculdade de Medicina da Universidade de São Paulo - USP - São Paulo (SP) - Brasil.

Médico Assistente Doutor do Departamento de Oftalmologia do Hospital das Clínicas da USP - São Paulo (SP) - Brasil.

Médica Assistente Doutora do Departamento de Oftalmologia do Hospital das Clínicas da USP - São Paulo (SP) - Brasil.

Oftalmologista do Departamento de Oftalmologia do Hospital das Clínicas da USP - São Paulo (SP) - Brasil. Oftalmologista do Departamento de Oftalmologia do Hospital das Clínicas da USP - São Paulo (SP) - Brasil.

Endereço para correspondência: Otacílio de Oliveira Maia Júnior. Rua Machado Bittencourt, 29 - Apto. 53 São Paulo (SP) CEP 04044-000

E-mail: omaiausp@uol.com.br

Recebido para publicação em 29.03.2005

Última versão recebida em 27.10.2005

Aprovação em 09.05.2006

Os autores declaram não possuir nenhum interesse financeiro neste estudo.

Nota Editorial: Depois de concluída a análise do artigo sob sigilo editorial e com a anuência do Dr. João J. Nassaralla Jr sobre a divulgação de seu nome como revisor, agradecemos sua participação neste processo.

INTRODUÇÃ̃O

A telangiectasia retiniana é uma anomalia vascular, caracterizada por dilatação irregular e incompetência da parede dos vasos ${ }^{(1)}$. O termo telangiectasia justafoveal idiopática (TJI) foi introduzido por Gass \& Oyakawa em $1982^{(2)}$ e posteriormente atualizado por Gass \& Blodi, em $1993^{(3)}$. Compreende o tipo 1, considerado uma variação da doença de Coats, que é dividido em 1A e 1B. A forma 1B é localizada e mais leve que a 1A. Os tipos 2 e 3 são considerados afecções adquiridas, com características peculiares ${ }^{(4-5)}$.

A TJI tipo 1A é uma alteração congênita, raramente bilateral, que acomete, predominantemente, o adulto jovem masculino, sem alterações sistêmi$\operatorname{cas}^{(3)}$. A telangiectasia é, usualmente, localizada na região temporal da mácula, afetando mais de dois diâmetros papilares e abrangendo a porção superior e inferior da rafe horizontal.

O edema de mácula, devido à exsudação, é a principal causa de perda visual nesses pacientes. A acuidade visual (AV) pode variar de 20/40 a 20/200. Um número limitado de casos de melhora da AV após tratamento com fotocoagulação a laser, na área da telangiectasia, foi relatado ${ }^{(2,6)}$. Em casos selecionados, a fotocoagulação com laser de argônio pode ser considerada, embora resolução espontânea possa ocorrer ${ }^{(2,7)}$.

Recentemente, relatos de TJI tratadas com acetato de triancinolona intravítrea obtiveram regressão do edema de mácula e melhora da $\mathrm{AV}^{(8-9)}$. 


\section{MÉTODOS}

Há aproximadamente cinco anos, um caso de telangiectasia retiniana justafoveolar idiopática tipo $1 \mathrm{~A}$ vem sendo acompanhado no Departamento de Oftalmologia do Hospital das Clínicas da Faculdade de Medicina da Universidade de São Paulo (HC-FMUSP). Em razão de mostrar-se refratário ao tratamento prévio com fotocoagulação a laser de argônio, o paciente foi submetido à injeção intravítrea de $4 \mathrm{mg}$ de acetato de triancinolona, conforme a técnica recomendada pelo "guideline", publicado recentemente ${ }^{(10)}$. Para tal, o paciente assinou termo de consentimento livre e esclarecido.

$\mathrm{O}$ efeito terapêutico foi demonstrado por meio da acuidade visual (medida pela tabela de Snellen) e da tomografia de coerência óptica (OCT). A angiofluoresceinografia prévia ao tratamento foi apresentada. As imagens obtidas pelo OCT-3 antes da injeção, na $3^{\underline{a}}$ e na $6^{\underline{a}}$ semana após tratamento, foram centradas na depressão foveal. Utilizando o programa de medida da

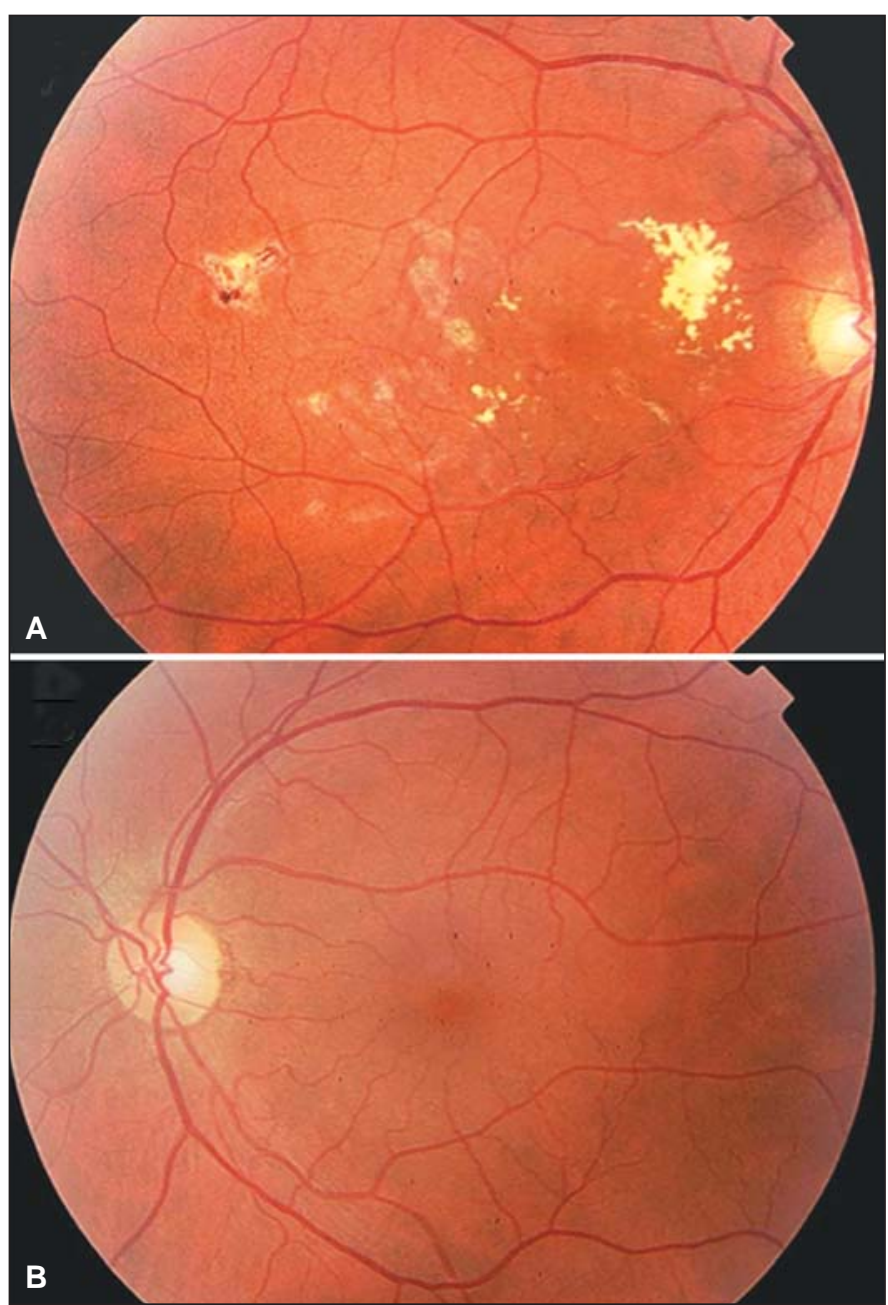

Figura 1 - Retinografia. A: Olho direito com cicatrizes da fotocoagulação na região paramacular temporal, telangiectasias justafoveais e exsudatos lipídicos residuais que acometem a mácula nasal e temporal; B: Olho esquerdo sem alterações. espessura da retina (Retinal thickness), o cursor foi colocado manualmente no centro da fóvea quando a depressão foveal era visível ou na parte mais espessa no centro da mácula.

\section{RELATO DE CASO}

MCR, 48 anos, sexo masculino, branco, comerciante, natural e procedente de São Paulo, com diagnóstico de telangiectasia retiniana justafoveolar tipo $1 \mathrm{~A}$ e submetido a três sessões de fotocoagulação a laser no período de 5 anos, sem apresentar melhora.

Ao exame, apresentava acuidade visual com melhor correção no olho direito $(O D)=20 / 100$ e no olho esquerdo $(O E)=20 / 20$, pressão intraocular (PIO) de $12 \mathrm{mmHg}$ no OD e de $14 \mathrm{mmHg}$ no OE e na biomicroscopia sem alterações em ambos os olhos (AO).

A fundoscopia do OD mostrava saculações dos capilares justafoveal, exsudatos e lesões hipercrômicas temporal à fóvea devido à fotocoagulação (Figura 1A), no OE não foi observada alteração (Figura 1B). Na angiofluresceinografia, observou-se uma hiperfluorescência perifoveal no OD, que aumentava de intensidade no decorrer do exame, por vazamento do contraste, além da hiperfluorescência circundando áreas hipofluorescentes das cicatrizes do laser (Figura 2). O OCT inicial evidenciou espessura macular de $569 \mu \mathrm{m}$ na área central e áreas de atrofia do epitélio pigmentado retiniano (Figura 3A).

O paciente foi submetido a uma aplicação de $4 \mathrm{mg}$ de triancinolona intravítrea no OD sem intercorrência. No exame da $3^{\underline{a}}$ semana, observou-se acuidade visual de 20/60 neste olho, com diminuição na espessura macular em relação à medida prétratamento: $371 \mu \mathrm{m}$ (Figura 3B). Após seis semanas ocorreu recidiva do edema, com piora na acuidade visual para 20/100 e aumento da espessura macular para $614 \mu \mathrm{m}$ (Figura 3C).

O paciente vem sendo avaliado periodicamente, com exame oftalmológico estável. Não foi detectada qualquer complicação pelo uso da triancinolona, a PIO variou de 12 a 14 mmHg durante as seis semanas de seguimento.

\section{DISCUSSÃO}

A TJI tipo 1A, considerada uma variante da doença de Coats, situa-se na região perifoveal, e a diminuição da AV se dá pelo edema macular ${ }^{(2-4)}$. O acúmulo de líquido nas camadas da retina é conseqüente à transudação a partir da parede dos vasos incompetentes. A histopatologia desses vasos mostra, através da microscopia eletrônica, além do espessamento da parede dos vasos, a presença de elementos figurados intramurais, principalmente hemácias, indicando anormalidade do endotélio, que apresenta a permeabilidade aumentada, devido a uma anomalia de desenvolvimento ${ }^{(11)}$.

A evolução dessa doença mostra, ao lado de raros casos com involução espontânea, a instalação de edema macular crônico com conseqüentes alterações estruturais irreversíveis na retina ${ }^{(3)}$. 
O tratamento proposto é a fotocoagulação dos vasos telangiectásicos, o que nem sempre é possível, devido à proximidade dos mesmos ao centro da fóvea. Pelo angiograma do presente caso (Figura 2), podem ser observadas as marcas de laser na região temporal da mácula com telangiectasias residuais ao redor da zona avascular da fóvea. A fotocoagulação desses vasos é tecnicamente difícil ou inviável.

$\mathrm{O}$ acetato de triancinolona é um potente esteróide que vem sendo utilizado amplamente em diversas doenças oftalmológicas, apresentando bons resultados ${ }^{(12-14)}$. É uma solução hidrofóbica, relativamente insolúvel, que parece manter níveis terapêuticos no vítreo por mais de três meses ${ }^{(15)}$. A toxicidade e farmacocinética da injeção intravítrea do acetato de triancinolona foram previamente analisadas em estudos com animais, mostrando-se seguras ${ }^{(16-17)}$. As complicações intravítreas mais comuns podem ser atribuídas ao próprio procedimento
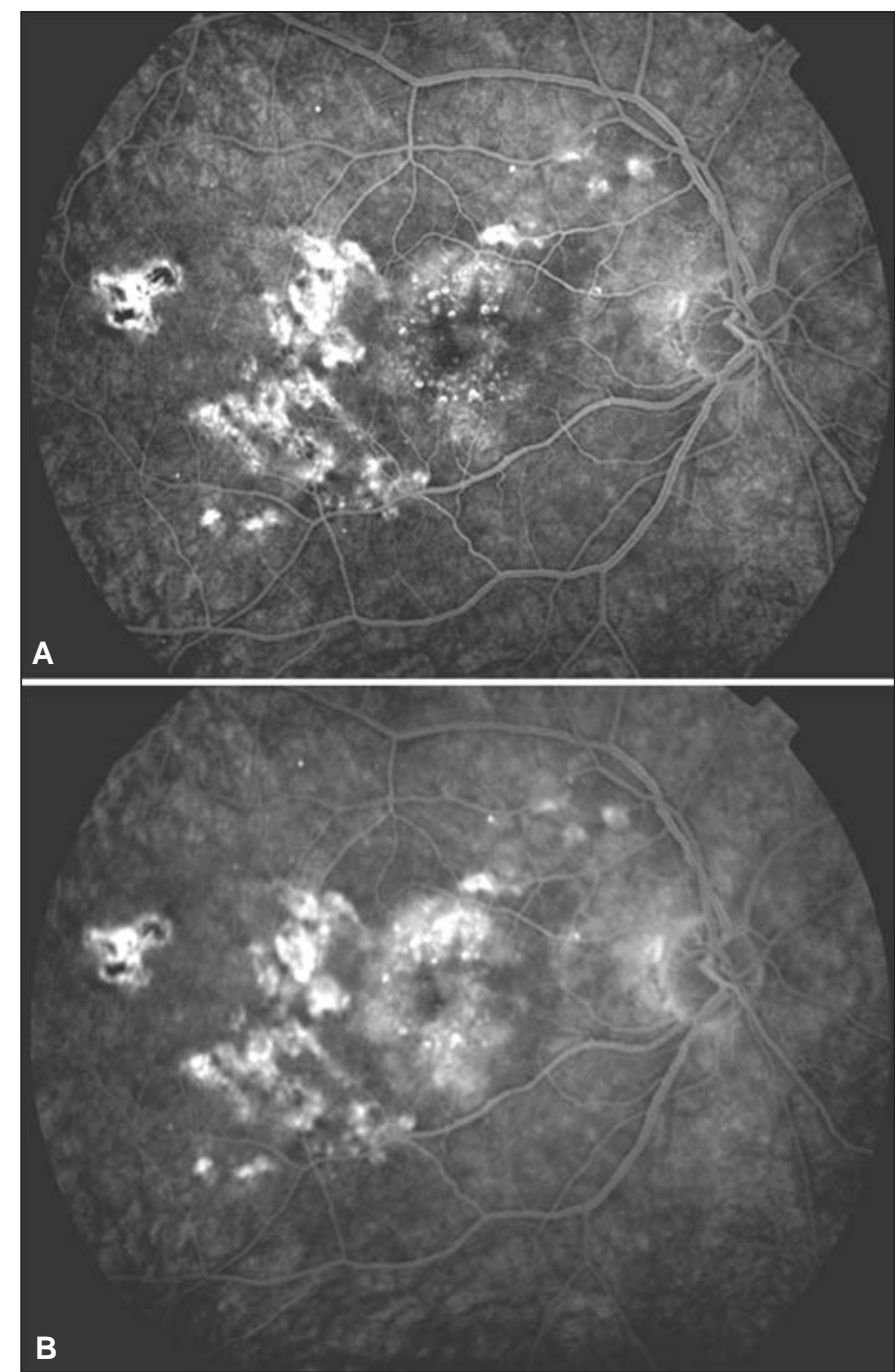

Figura 2 - Angiofluoresceinografia do olho direito. A: Fase arteriovenosa individualizando as telangiectasias residuais ao redor da zona avascular da fóvea; B: Fase tardia mostrando extravasamento do corante nesta região. da injeção ou aos efeitos colaterais dos esteróides, como catarata e glaucoma ${ }^{(10,15)}$. O uso desse medicamento intravítreo, apesar do baixo índice de complicação inerente à substância, aliado a uma boa técnica de aplicação, não é totalmente inócuo $^{(10,15)}$. Portanto, deve-se avaliar cuidadosamente a indicação desse esteróide.

A estabilização da barreira hematorretiniana pode ser obtida com uso da triancinolona intravítrea ${ }^{(18)}$. Como na TJI tipo 1A existe um aumento da permeabilidade vascular pelas alterações telangiectásicas, pode-se restabelecer essa barreira com esteróide intravítreo, diminuindo o edema macular e podendo melhorar a acuidade visual. Recentemente, o uso de acetato de triancinolona intravítrea foi introduzido no arsenal terapêutico da TJI, demonstrando melhora após o tratamento $^{(8-9)}$. Os dois casos relatados na literatura são de TJI tipo 2, ou seja, adquirida e bilateral. No primeiro relato, a acuidade visual melhorou de 20/80 para 20/60 em três semanas após a injeção, persistindo nesse nível até o final do follow-up de oito semanas ${ }^{(8)}$. No segundo relato, no qual a visão melhorou de 20/60 para 20/25 após injeção, num seguimento de seis
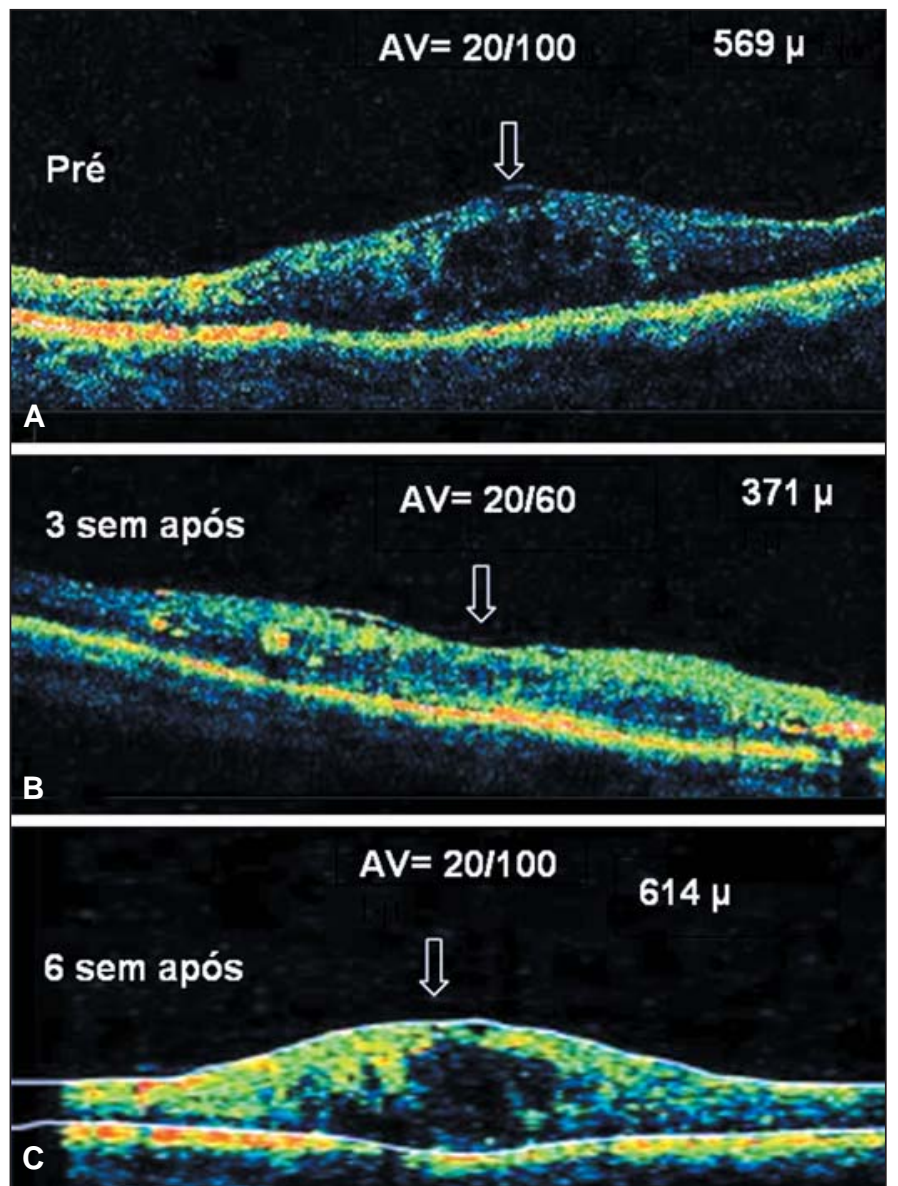

Figura 3 - "Scan" horizontal do OCT de $6 \mathrm{~mm}$ centrado na fóvea. A: antes da injeção intravítrea de $4 \mathrm{mg}$ de triancinolona, espessura $569 \mu \mathrm{m}$ e $A V=20 / 100$; $B$ : três semanas após a aplicação, espessura $371 \mu \mathrm{m}$ e $A V=20 / 60$; $C$ : seis semanas após a aplicação, espessura de $614 \mu \mathrm{m}$ e $A V=20 / 100$ 
meses, parece haver outra doença concomitante à TJI. Segundo o angiograma apresentado pelo autor, antes do tratamento houve hiperfluorescência peripapilar no OE (tratado) em relação ao OD (não tratado); esse achado não faz parte do quadro da TJI do tipo 2. Após a reabsorção do edema macular cistóide, que também não faz parte do quadro desta doença, observam-se pontos de extravasamento de corante extramacular, indicando a presença de outra doença ${ }^{(9)}$.

No presente caso, houve diminuição do edema macular e melhora na acuidade visual após três semanas da aplicação intravítrea de triancinolona, porém após seis semanas houve recidiva do quadro. Na terceira semana após da injeção, a acuidade visual melhorou de 20/100 para 20/60, devido à absorção parcial do edema. O OCT da terceira semana mostrou espessura de $371 \mu$, ainda bem acima da espessura da mácula normal $^{(19)}$. Com a recidiva do edema, após seis semanas do procedimento, a acuidade visual voltou ao nível pré-tratamento e a espessura central ao OCT foi de $614 \mu \mathrm{m}$.

\section{CONCLUSÃO}

A estabilização da parede vascular obtida com injeção intravítrea de triancinolona proporciona uma melhora transitória da visão e do edema macular em olhos com TJI 1A. Não foi demonstrada nenhuma ajuda permanente à fotocoagulação prévia no tratamento desse caso.

\section{ABSTRACT}

Case report of one idiopathic juxtafoveal telangiectasis (IJT) 1A patient whose right eye was treated with a $4 \mathrm{mg}$ intravitreal triamcinolone acetonide injection. The outcome was evaluated by visual acuity and optic coherence tomography. The visual acuity and the caliper retinal thickness before triamcinolone injection were respectively $20 / 100$ and $569 \mu \mathrm{m}$, and 20/60 and $371 \mu \mathrm{m}$ after three weeks and 20/100 and $614 \mu \mathrm{m}$ after six week of follow-up. The stabilization of the vascular wall due to the intravitreal triamcinolone injection leads to a transitory improvement in vision and reduction in macular edema in the TJI 1A eyes. No permanent help by the photocoagulation could be shown.
Keywords: Fovea centralis/pathology; Fundus oculi; Retinal neovascularization; Telangiectasis/diagnosis; Triamcinolone/therapeutic use; Tomography, optical coherence; Case reports [publication type]

\section{REFERÊNCIAS}

1. Reese AB. Telangiectasis of the retina and Coat's disease. Am J Ophthalmol. 1956;42(1):1-8.

2. Gass JD, Oyakawa RT. Idiopathic juxtafoveolar retinal telangiectasis. Arch Ophthalmol. 1982;100(5):769-80.

3. Gass JD, Blodi BA. Idiopathic juxtafoveolar retinal telangiectasis. Update of classification and follow-up study. Ophthalmology. 1993;100(10):1536-46.

4. Chopdar A. Retinal telangiectasis in adults: fluorescein angiographic findings and treatment by argon laser. Br J Ophthalmol. 1978;62(4):243-50.

5. Park DW, Schatz H, McDonald HR, Johnson RN. Grid laser photocoagulation for macular edema in bilateral juxtafoveal telangiectasis. Ophthalmology. 1997; 104(11):1838-46

6. Alldredge CD, Garretson BR. Intravitreal triamcinolone for the treatment of idiopathic juxtafoveal telangiectasis. Retina. 2003;23(1):113-6.

7. Abujamra S, Bonanomi MT, Cresta FB, Machado CG, Pimentel SL, Caramelli CB. Idiopathic juxtafoveolar retinal telangiectasis: clinical pattern in 19 cases. Ophthalmologica. 2000;214(6):406-11.

8. Bonanomi MTBC, Cresta FB, Abujamra S. Telangiectasia retiniana justafoveolar idiopática do grupo 2A: hiperpigmentação macular incomum e dois pacientes. Arq Bras Oftalmol. 2001;64(6):569-72.

9. Tripathi R, Ashton N. Electron microscopical study of Coat's disease. Br J Ophthalmol. 1971;55(5):289-301.

10. Challa JK, Gillies MC, Penfold PL, Gyrory JH, Hunyor AB, Billson FA. Exudative macular degeneration and intravitreal triamcinolone: 18 month follow up. Aust N Z J Ophthalmol. 1998;26(4):277-81.

11. Martidis A, Duker JS, Greenberg PB, Rogers AH, Puliafito CA, Reichel E, Baumal C. Intravitreal triamcinolone for refractory diabetic macular edema. Ophthalmology. 2002;109(5):920-7.

12. Andrade RE, Muccioli C, Farah ME, Nussenblatt RB, Belfort R Jr. Intravitreal triamcinolone injection in the treatment of serous retinal detachment in Vogt-Koyanagi-Harada syndrome. Am J Ophthalmol. 2004;137(3):572-4.

13. McCuen BW 2nd, Bessler M, Tano Y, Chandler D, Machemer R. The lack of toxicity of intravitreally administered triamcinolone acetonide. Am J Ophthalmol. 1981;91(6):785-8.

14. Schindler RH, Chandler D, Thresher R, Machemer R. The clearance of intravitreal triamcinolone acetonide. Am J Ophthalmol. 1982;93(4):415-7.

15. Peyman GA, Moshfeghi DM. Intravitreal triamcinolone acetonide. Retina. 2004;24(3):488-90.

16. Martinez JA. Intravitreal triamcinolone acetonide for bilateral acquired parafoveal telangiectasis. Arch Ophthalmol. 2003;121(11):1658-9.

17. Wilson CA, Berkowitz BA, Sato Y, Ando N, Handa JT, de Juan E Jr. Treatment with intravitreal steroid reduces blood-retinal barrier breakdown due to retinal photocoagulation. Arch Ophthalmol. 1992;110(8):1155-9.

18. Aiello LP, Brucker AJ, Chang S, Cunningham ET Jr, D'Amico DJ, Flynn HW Jr, et al. Evolving guidelines for intravitreous injections. Retina. 2004; 24(5 Suppl):S3-19.

19. Hee MR, Puliafito CA, Duker JS, Reichel E, Coker JG, Wilkins JR, et al Topography of diabetic macular edema with optical coherence tomography. Ophthalmology. 1998;105(2):360-70. 